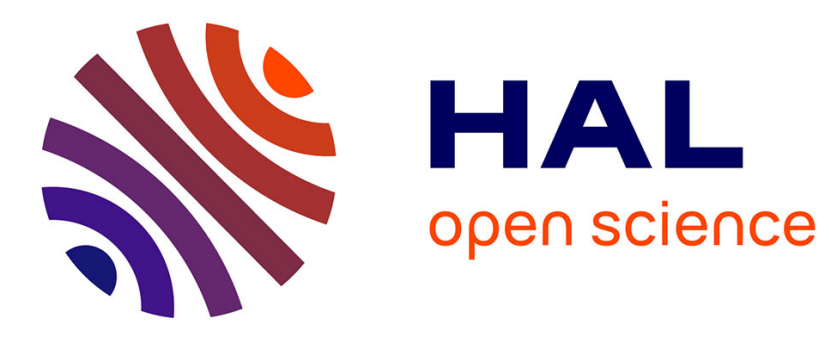

\title{
Detection of Defects in Malus asiatica Nakai Using Hyperspectral Imaging
}

\author{
Jianglong Liu, Shujuan Zhang, Haixia Sun, Zhiming Wu
}

\section{To cite this version:}

Jianglong Liu, Shujuan Zhang, Haixia Sun, Zhiming Wu. Detection of Defects in Malus asiatica Nakai Using Hyperspectral Imaging. 10th International Conference on Computer and Computing Technologies in Agriculture (CCTA), Oct 2016, Dongying, China. pp.111-122, 10.1007/978-3-03006155-5_11. hal-02179993

\section{HAL Id: hal-02179993 \\ https://inria.hal.science/hal-02179993}

Submitted on 12 Jul 2019

HAL is a multi-disciplinary open access archive for the deposit and dissemination of scientific research documents, whether they are published or not. The documents may come from teaching and research institutions in France or abroad, or from public or private research centers.
L'archive ouverte pluridisciplinaire HAL, est destinée au dépôt et à la diffusion de documents scientifiques de niveau recherche, publiés ou non, émanant des établissements d'enseignement et de recherche français ou étrangers, des laboratoires publics ou privés.

\section{(c)(1)}

Distributed under a Creative Commons Attribution| 4.0 International License 


\title{
Detection of Defects in Malus asiatica Nakai Using Hyperspectral Imaging
}

\author{
Jianglong Liu, Shujuan Zhang ${ }^{(\bowtie)}$, Haixia Sun, Zhiming Wu \\ Department of Engineering, Shanxi Agricultural University, Taigu 030801, China \\ longxing_815@163.com, zsujuan@263.net, sunhaixial121@126.com, \\ zhim_wu@163.com
}

\begin{abstract}
Hyperspectral imaging technology was employed to detect defects such as rot, bruise and rust in Malus asiatica Nakai. 213 RGB images of samples, including 3 types of damage samples and sound ones, were acquired by hyperspectral imaging system. Spectral data were extracted from the regions of interest (ROI) using ENVI4.7 software. Competitive adaptive reweighted sampling (CARS) and successive projections algorithm (SPA) were used to select characteristic wavelength points. As a result, 11 and 6 characteristic wavelength points were chosen for CARS and SPA respectively. Extreme learning machine (ELM) discrimination model was established based on the spectral data of selected wavebands. The results showed that the accuracy of the SPA-ELM discrimination model was as great as $94.74 \%$. Then, images corresponding to six sensitive bands $(532 \mathrm{~nm}, 563 \mathrm{~nm}, 611 \mathrm{~nm}, 676 \mathrm{~nm}, 812$ $\mathrm{nm}$, and $925 \mathrm{~nm}$ ) selected by SPA were selected for principal components analysis (PCA). Finally, the images of PCA were employed to identify the location and area of a defect's feature through imaging processing. Through sobel operator and region growing algorithm, the edge and defective feature of 38 Malus asiatica Nakai can be recognized and the detection precision was $92.11 \%$. This study demonstrated that the defects, (rot, bruise, and rust) of Malus asiatica Nakai can be detected in spectral analysis and feature detection in hyperspectral imaging technology, which provides a theoretical reference for the online detection of defects in Malus asiatica Nakai.
\end{abstract}

Key words: Malus asiatica Nakai; Defects; Hyperspectral imaging; Detection

\section{Introduction}

Malus asiatica Nakai, Chinese pearleaf crabapple, is a species in the genus Malus which belongs to the family of Rosaceae. It is widely distributed in the Yellow River region, the Yangtze River basin and in Liaoning province, and has a long cultivation history of thousands of years. Malus asiatica Nakai is unique to China. Malus asiatica Nakai's fruit is rich in various nutrients, including iron, zinc, selenium and other trace elements as well as vitamins which are essential to the human body [1]. It is known for its medicinal effect in treating cough asthma, coronary heart disease and high blood pressure. The fruit also has the very high value with the medicine. Malus asiatica Nakai can be eaten directly or can be made into other products such as preserved fruit, beverages, etc. Because of these factors and low prices, this fruit is quite popular with 
consumers.

However, defective tissue can seriously affect the appearance of the fruit, and releases ethylene, which damages the overall quality of the fruit. Defective tissue can also influence storage and shelf life of fresh fruits [2]. The defects include damage to the skin and internal organization in natural conditions and transportion. This paper focuses on three types of common defects, (rot, bruise, and rust) of Malus asiatica Nakai and helps ascertain methods to identify them. Rot was formed due to natural factors such as wind, rain and insects, which potentiate the invasion and destruction of bacteria. During harvesting, packaging and transportation, the major damage to the fruit is usually bruising which is caused by impact from external forces and squeezing; Rusty spots, also known as fruit rust, is a layer of yellow tan plaques with irregular shape and different sizes, which is a kind of physiological disease on the surface of the skin. It is a biological protective response that occurs when epidermal cells meet adversity such as low temperature and polluted air. Rapid and nondestructive testing of Malus asiatica Nakai would have a meaningful impact in selecting defective fruits. By employing hyperspectral imaging technology, 3 types of defective and sound samples were identified in this study.

Hyperspectral imaging technique is a technology that integrates spectrum and images into one system, and has been widely used in nondestructive detection of agricultural products [3]. It performs a kind of hyperspectral analysis of spectrum and images to identify and classify damaged samples and to find a correlation between spectral characteristics and physico-chemical properties of affected samples.

Combining hyperspectral imaging technology and minimum noise fraction, Baohua Zhang [4] detected slight damages in Fuji apples. The result showed that the correct recognition rate was $97.1 \%$. Jiewen Zhao [5] implemented a correct recognition rate of $88.57 \%$ for slight damages in apples by using a principal component analysis (PCA) method to get the image under the characteristic wavelength of $547 \mathrm{~nm}$ based on hyperspectral imaging. Baranowski [6] studied apples using hyperspectral imaging in 2001, the principal components analysis (PCA) of the images captured in broad spectrum ranges (400-2500) made it possible to distinguish between early bruises and the sound ones. Lee [7] applied hyperspectral imaging technology to detect bruises in Shingo pears, and the results showed that the recognition rate was $92 \%$. In order to detect damages to kiwi fruit, Qiang Lv [8] used hyperspectral imaging and parallelepiped classification. Four principal component images were found by processing principal components analysis (PCA) based on the band data from 600$1000 \mathrm{~nm}$, and the results showed that the overall recognition rate was $85.5 \%$. ByoungKwan Cho [9] also found cuticle defects in cherry tomatoes by hyperspectral fluorescence imagery with a correct classification greater than 99\%. Thus hyperspectral imaging has been used frequently and with success to detect fruit quality. This study is the first to use hyperspectral imaging technology to detect the diversified defects in Malus asiatica Nakai.

The goal of this paper was to study the potential for hyperspectral image on three kinds of defects of Malus asiatica Nakai. Specific aims are as follows:

By employing hyperspectral imaging technology, both image information of defective fruits and sound samples were acquired.

Competitive adaptive reweighted sampling (CARS) and successive projections algorithm (SPA) were used to extract characteristic wavelength points.

The information obtained based on characteristic wavebands was used to build detection models, the extreme learning machine (ELM) and the image progressing algorithm, from two aspects of spectrum and images respectively. 


\section{Materials and methods \\ 2.1 Experimental materials}

The sample's material used in the study came from the same orchard in Xiaobai county, Taigu city. There were a total of 213 fruits used, including 58 rotten samples, 56 bruised samples and 39 rusted samples and 60 sound ones, which were collected directly after being harvested by hand in 2015 , and were then stored at $21^{\circ} \mathrm{C}$. The fruits were selected in a regular pattern and only fruits with an equatorial diameter between $27.50 \mathrm{~mm}-30.00 \mathrm{~mm}$ and a mass between $17.40 \mathrm{~g}-20.50 \mathrm{~g}$ were studied.

\subsection{Instrumentation}

Image information for all samples was collected using a hyperspectral imaging system as shown in fig. 1. Visible and near infrared (VNIR) ImSpector V10E imaging spectrograph (380nm-1030nm) was manufactured by Specim Finland. Two EKE-ER 500W halogen lamps were used for illumination, and a charge coupled device was used to achieve signal conversion. Other auxiliary equipment was included in this system, such as an OLES23 camera lens, an electronically controlled platform and a computer. By employing this system, Malus asiatica Nakai can be scanned by being moved through the field of view on the spectrograph's platform. In the process of gathering samples' spectral information parameters such as setting a reasonable exposure time and platform moving speed were important. After several studies and tests, the camera exposure time was set to $0.09 \mathrm{~s}$ and platform's moving speed was set to $2.58 \mathrm{~mm} / \mathrm{s}$. In order to obtain the desired spectrum of samples, the target area was positioned toward the lens, and was $310 \mathrm{~mm}$ away from the equatorial plane of the fruit. The distinguishability of images acquired by this system was $672 * 512$ pixels.

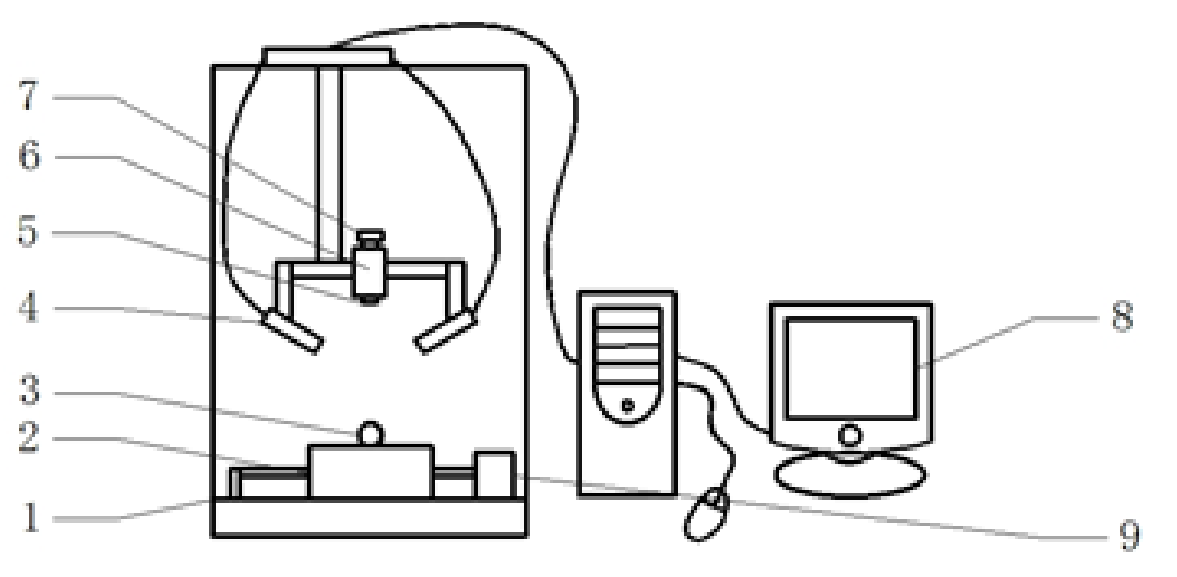

1. Dark chamber 2. Mobile platform 3. Sample 4. Illumination 5. Lens

6. Spectrograph 7. Camera 8. Computer 9. Motor

Fig. 1. Schematic diagram of hyperspectral imaging system

\subsection{Spectral data processing and analysis software}


Spectral-cube data acquisition V10 software (Isuzu Optics Corpfrom, Taiwan) was applied to set parameters for collection of spectra. Regions of Interest (ROI) were extracted through ENVI4.7 (ITT Visual Inforamtion Solutions, USA). Matlab R2012a software (The Math Works, Natick, USA) was applied in building recognition models. Some data was processed by The Unscramber X 10.1 (CAMO AS, Oslo, Norway) and other softwares.

\subsection{Spectrum collecting}

Given the spherical shape of Malus asiatica Nakai, light is distributed from the bright center to the dark edge, so the spectrum of the defective area was collected in same position to reduce the impact of uneven illumination intensity for spectrum. Regions of Interest (ROI) from defective and sound samples were extracted through ENVI4.7 software with a resolution of 5 (spatial) by 5 (spectral) pixels. After that, the average spectrum data was calculated. Bands containing noise were removed, and only the bands between $480-1000 \mathrm{~nm}$ were used in the following study.

\subsection{Calibration method of hyperspectral image}

Because of dark electric current in the camera, differences existing in the light source under the uneven intensity of each band and other factors, the results were affected by noise thereby affecting the accuracy of the model [10]. The raw images from the cameras were calibrated to obtain the reflectance $\mathrm{R}$ by using equation 1 , which helped eliminate the effects of noises.

$$
R=\frac{I_{i m}-I_{d}}{I_{w}-I_{d}}
$$

Where $\mathrm{R}$ is the intensity of the calibrated image, Iim is the intensity of any raw image; and Id is the intensity of the dark image when the lens was covered with a black cap and the lamp was turned off; Iw is the intensity of the white reference Spectralon plate.

\subsection{Analyzing algorithms}

Two methods were used to select characteristic wavelengths and reduce the dimensionality of the data sets, which were then used to get useful information about fruit defects. The first method was competitive adaptive reweighted sampling (CARS) [11], which is a method that simulates the mechanism of survival of the fittest in Darwin's evolution theory. Wavelength points with large absolute values of regression coefficients were kept and smaller ones were removed by using a Partial Least Squares (PLS) model. Cross validation (CV) was applied as a way to select the subsets with the lowest values of standard error of cross validation. Allowing for the effective selection of optimal wavebands that carried useful information related to properties. The other method to select characteristic wavelengths was successive projections algorithm (SPA) [12], which finds groups containing a minimum of redundant information from a variable of full spectral information. The effect of multicollinearity among variable wavelengthes was eliminated or minimized through 
SPA, which reduced the complexity of the data. SPA achieved excellent results in selecting wavelengths for variety of samples.

To compress the data and enhance image information of Hyperspectral image, principal component analysis (PCA) [13] was carried out as an image pre-processing method in which useful information about fruit defects was found. By applying SPA, a new dimension of relevant variables replaced the original correlated variables through a linear combination. The new variables, which contained information related to a defect's features, called principal component (PC). However, only the first few $\mathrm{PC}$ bands contained the majority of relevant information.

In this study the extreme learning machine (ELM) [14] was applied for pattern recognition. This method was chosen because of its excellent learning ability, good generalization, simple parameter setting. ELM's hidden node parameter was generated and then used the weight that was found from outside to determine the output result, which greatly simplified the iterative process compared with traditional neural networks. Exciting function and hidden node parameters needed to be determined before building the ELM model. In this paper, function as activating was Sigmoidal and the initial value of node parameter was 14 .

In this study, sobel operator and region growing algorithm are powerful methods to recognize the edge and defect feature for image information.

\section{$3 \quad$ Results and discussion}

\subsection{Discrimination of defects in Malus asiatica Nakai based on spectra}

\subsubsection{The average spectral curves of Malus asiatica Nakai}

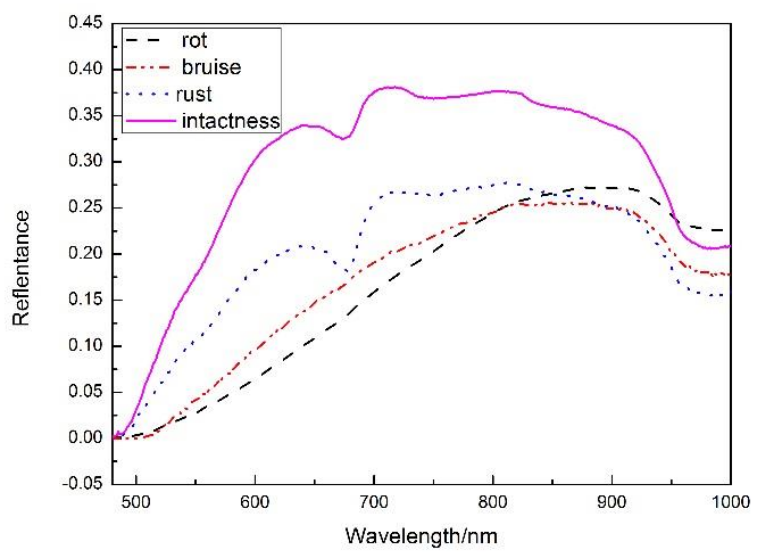

Fig.2. Average spectral curves of four different Malus asiatica Nakai samples

The mean original spectra response in defective and intact samples is presented in fig.2. As shown in picture, the spectral curve of sound samples had intense absorption bands at $680 \mathrm{~nm}$ and $970 \mathrm{~nm}$. The intense absorption band at $680 \mathrm{~nm}$ was caused by absorption of chlorophyll and reflection of color information on the fruit surface [15]. 
While the absorbance peak at 970nm (the second overtone of the O-H played a dominant role) associated with water, which dictated the height of absorption peak [16]. The rotten and bruised areas had a lower reflectance at bands from $450 \mathrm{~nm}$ to $800 \mathrm{~nm}$, and the absorbance peak at 680 was weak because the dark epiderm in defects had a lower reflectance than a peel in a sound region.

\subsubsection{Extracting characteristic wavelengths}

As mentioned in section 2, two methods were programmed by Matlab R2012a software to extract characteristic wavelengths. The result found in CARS is displayed in fig 3 . The curve marked with (a) in fig 3 is the variation tendency of the number of waves in the progress of selecting bands. Fig. 3(b) shows the trends of the curves about the root mean squares error of cross validation (RMSECV). The RMSECV' curve showed a rising trend because the first 34 sifting processes excluded bands that had nothing to do with detection of defects. However, the value of RMSECV increased after 34 th selection because the important variables were removed. The trend of the regression coefficient for each wavelength is shown in fig. 3(c). As a result, 11 characteristic wavebands were chosen, and were located at $515 \mathrm{~nm}, 532 \mathrm{~nm}$, $541 \mathrm{~nm}, 560 \mathrm{~nm}, 566 \mathrm{~nm}, 715 \mathrm{~nm}, 920 \mathrm{~nm}, 931 \mathrm{~nm}, 942 \mathrm{~nm}, 988 \mathrm{~nm}$ and $998 \mathrm{~nm}$. Fig. 4 shows the results found through SPA. RMSE distribution of SPA in different variables is displayed in fig. 4(a), and the picture marked with $\mathrm{b}$ in fig.4 shows the optimum characteristic wavebands selected by SPA. It can be derived from fig. 4 that the root mean square error reached a minimum when the number of selecting wavebands was 6 . Thus six wavebands at $563 \mathrm{~nm}, 925 \mathrm{~nm}, 812 \mathrm{~nm}, 532 \mathrm{~nm}, 611 \mathrm{~nm}$ and $676 \mathrm{~nm}$ were selected. The importance is reflected in descending order.

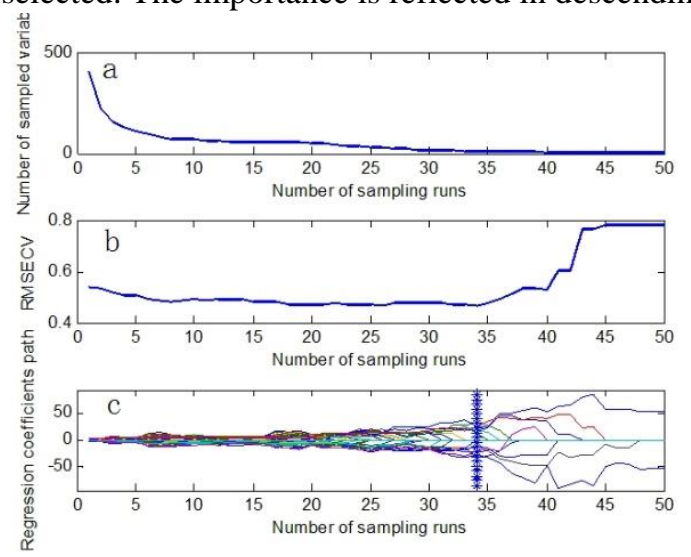

Fig.3. Result of variable selection on Malus asiatica Nakai 


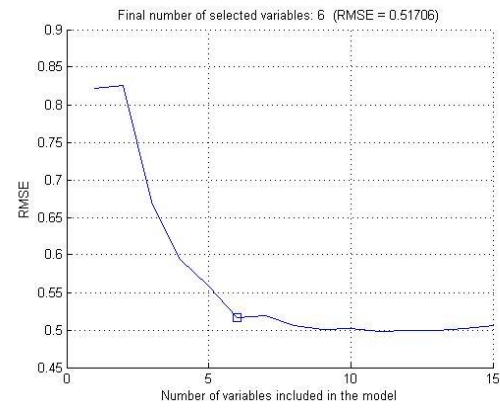

(a)RMSE distribution of SPA for different variables

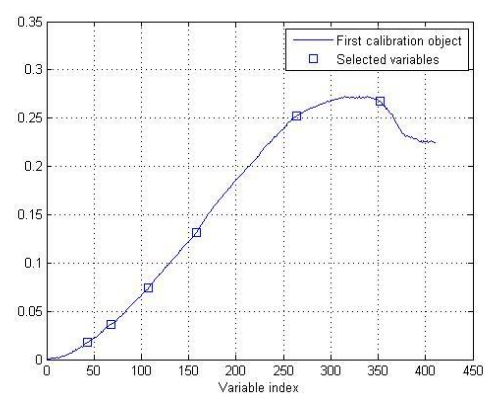

(b) Optimum characteristic wavebands selectded by SPA

Fig.4. RMSE distribution of SPA for different variables and optimum characteristic wavebands selectded by SPA

\subsubsection{ELM identification model for defects of Malus asiatica Nakai}

It is essential to decide the initial value for the node parameter in order to improve the accuracy of ELM. As is mentioned in the last section, the numbers below 159, the value of samples in the calibration set, were tested with a hidden node parameter with a step of 14 to find the proper node parameter. Results illustrated that 28 has the highest recognition rate, and is the best choice for the node parameter. The extracted characteristic wavebands were found using two different methods, and were then set as independent variables. Defective class codes were set as dependent variables. Independent variables could be randomly divided into the training set and the test set around the proportion of 3:1 by ELM. The performance of ELM discrimination models are presented in fig. 5 and fig. 6 separately. The CARS-ELM's success rate for rot was $93.75 \%$, bruise was $85.00 \%$, rust was $100 \%$, and sound was $100 \%$ and the total score is $92.98 \%$. The SPA-ELM model's success rate for rot was $94.11 \%$, bruise was $90 \%$, rust was $100 \%$, sound was $100 \%$ and the total score is $94.74 \%$. It could be found that the SPA-ELM model performs better on the classification of defective and sound Malus asiatica Nakai. But both two models have a lower accuracy for bruises. 


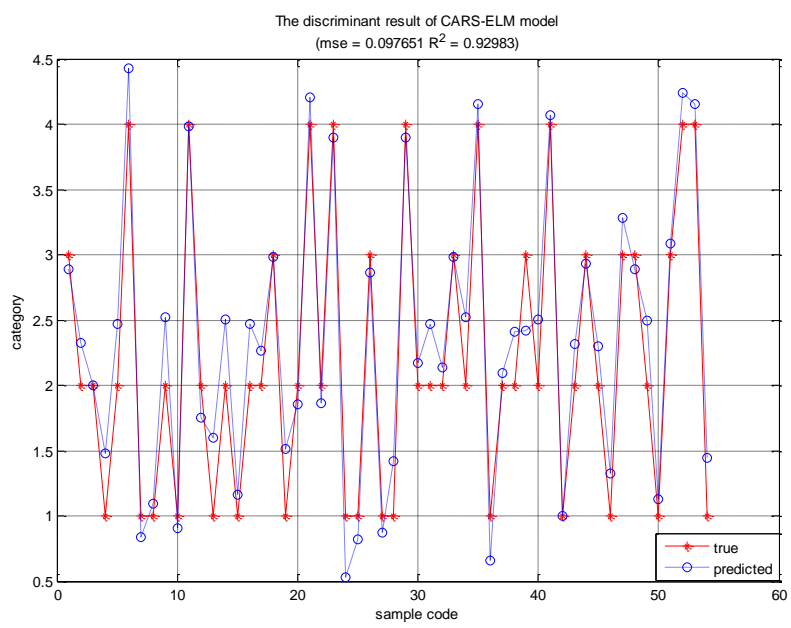

Fig.5. The discriminant result of CARS-ELM model

For category, where code 1 is the rot, code 2 is the bruise, code 3 is the bruise, and code 4 is the sound The discriminant result of SPA-ELM mod

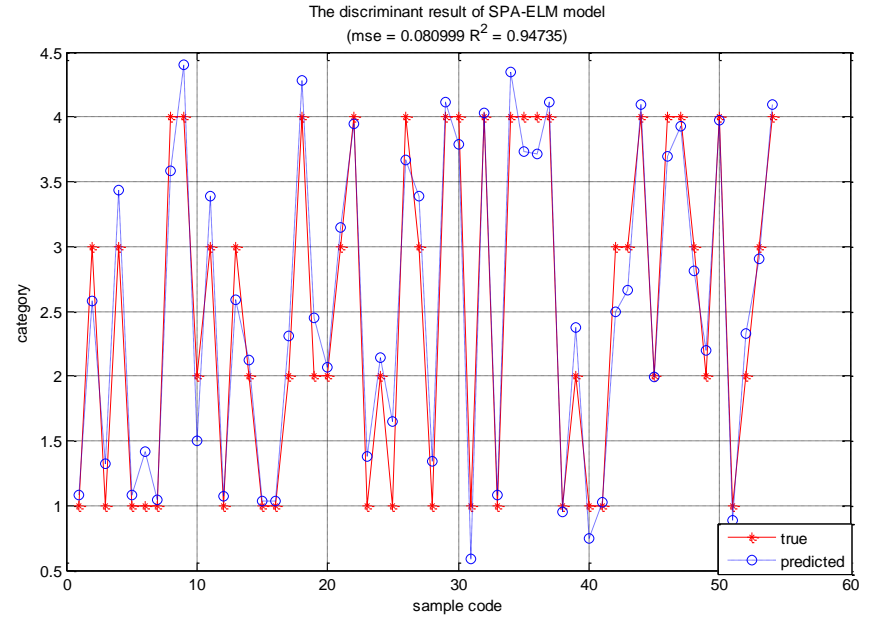

Fig.6. The discriminant result of SPA-ELM model

For category, where code 1 is the rot, code 2 is the bruise, code 3 is the bruise, and code 4 is the sound

\subsection{Discrimination of defects for Malus asiatica Nakai based on image progress-} ing

The results of Section 3.1 demonstrate that the model, which uses bands extracted by the SPA method, have higher precision in detecting defects for Malus asiatica Nakai when using the images of 6 bands $(63 \mathrm{~nm}, 925 \mathrm{~nm}, 812 \mathrm{~nm}, 532 \mathrm{~nm}, 611 \mathrm{~nm}$, and 676 $\mathrm{nm}$ ) extracted by SPA analysed principal component. PCA was carried out to find the images that contain the main information and improve the efficiency of testing. The cumulative contribution rate of six principal components is presented in table 3 . The cumulative contribution rate of the first four PC image reached to $99.87 \%$, and the main component images of PC1-PC4 are shown in Fig.7, which were used for the 
following study.

Table 3. Cumulative contribution rate of six principal components

\begin{tabular}{ccc}
\hline PC & Eigenvalue & Percent \\
\hline 1 & 1244425.04 & $94.90 \%$ \\
2 & 54494.42 & $99.06 \%$ \\
3 & 7838.19 & $99.66 \%$ \\
4 & 2774.20 & $99.87 \%$ \\
5 & 1138.46 & $99.96 \%$ \\
6 & 564.14 & $100.00 \%$ \\
\hline
\end{tabular}

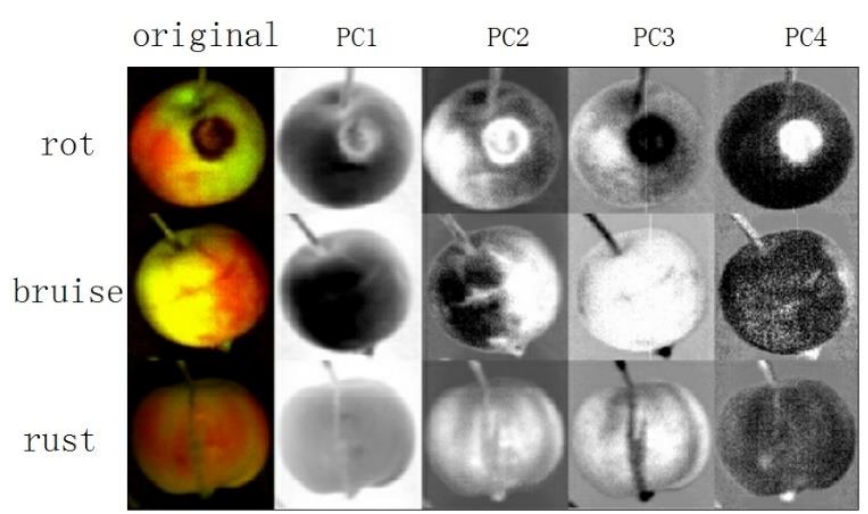

Fig.7. First four principal components grayscale of Malus asiatica Nakai samples

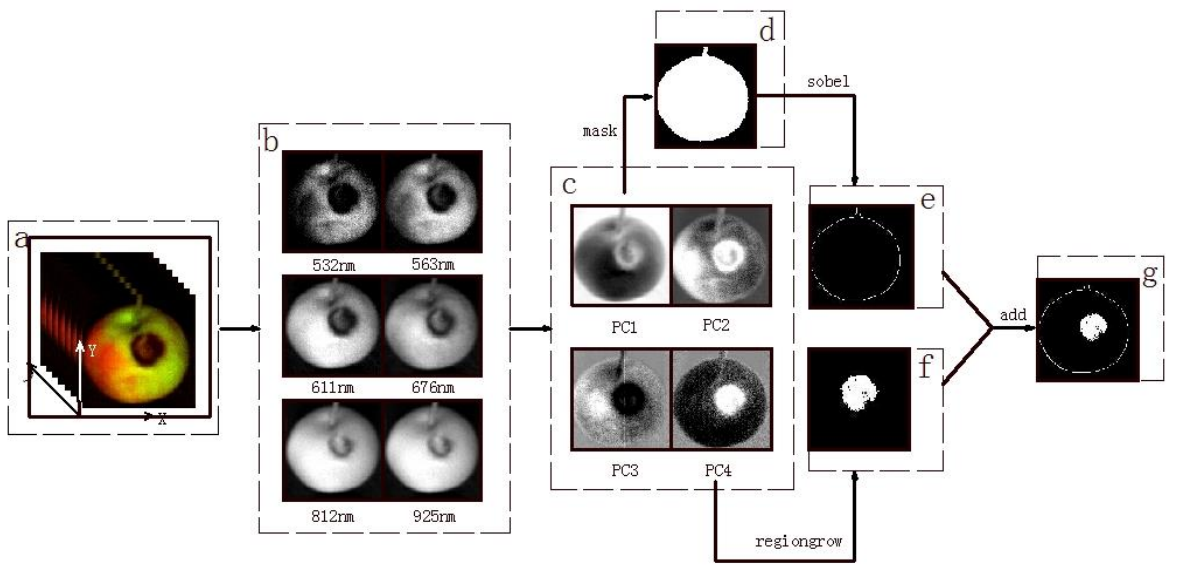

Fig.8. Flow chart of the key steps involved in defection algorithm about rot sample

As depicted in Fig. 8, the flow chart of the key steps is given about the defection algorithm. Rot for example goes from step (a) to (b), the images of 6 sensitive wavebands ( $63 \mathrm{~nm}, 925 \mathrm{~nm}, 812 \mathrm{~nm}, 532 \mathrm{~nm}, 611 \mathrm{~nm}$ and $676 \mathrm{~nm})$ were selected by SPA. Then analysed principal components of them were used to generate the picture (c), in which the main component image of PC1-PC4 is shown. In order to obtain the edge information of defective samples in Malus asiatica Nakai, the background image needs to be removed. The image of PC1 was executed to mask because of the maximum difference between target area and background. The sobel operator method 
was used to recognize the edge, which is displayed in image (e). Because of the obvious contrasts between the rot region and the sound area, the images of the PC4 were executed region growing algorithm method to get the information of rot area in recognition the rot of Malus asiatica Nakai [17]. The result is presented in image (f). As image ( $\mathrm{g}$ ) shows the final results of rot identification were found by adding images (e) and (f). According to the progress of image recognition in fig. 8, three defective samples of Malus asiatica Nakai in the prediction set were identified and the recognition of the effect is presented in fig. 9 and table 4.

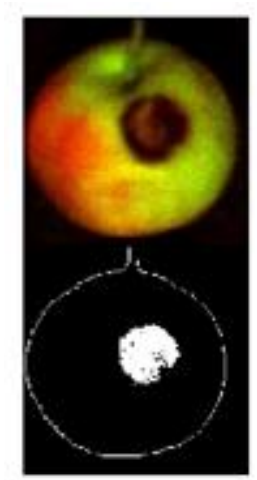

a

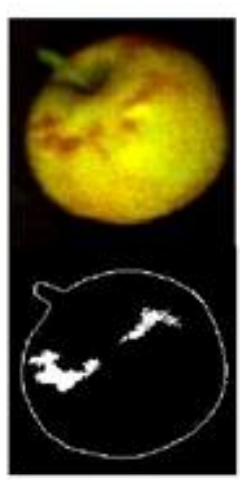

b

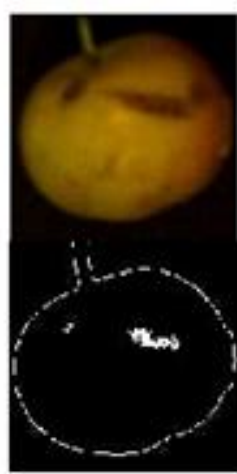

c

a.rot

b.bruise

c.rust

Fig.9. the identification of defects on Malus asiatica Nakai

Table 4. Detection results of defects in Malus asiatica Nakai

\begin{tabular}{cccc}
\hline $\begin{array}{c}\text { Malus asiatica } \\
\text { Nakai types }\end{array}$ & $\begin{array}{c}\text { sample } \\
\text { number }\end{array}$ & detected & Accuracy \\
\hline rot & 14 & 12 & $85.71 \%$ \\
bruise & 14 & 14 & $100 \%$ \\
rust & 10 & 9 & $90.00 \%$ \\
sum & 38 & 35 & $92.11 \%$ \\
\hline
\end{tabular}

The qualitative discrimination and qualitative identification of defects of Malus asiatica Nakai that employed image processing can achieve an accuracy of $92.11 \%$. Among 38 samples, 3 samples were not identified correctly. Two samples of rot were not properly identified. This can be attributed to the low degree of rotting decay. Similarly a sample's rust area was too small to be identified effectively.

Keqiang $\mathrm{Yu}$ [2] detected creak features in fresh jujubes by combining hyperspectra with image processing technology, the discrimination accuracy was $90.5 \%$. In the present study, three different defects in Malus asiatica Nakai were detected through spectrum technology and image technology. ELM discrimination model was applied based on characteristic wavebands. Bisides, image discrimination algorithms were applied to identify the defective features.

\section{Conclusions}

In the present study, defects of Malus asiatica Nakai were detected by employed 
spectrum technology and image processing technology respectively. The main conclusions can be drawn as follows:

The extreme learning machine (ELM) discrimination model was established by using the wavebands selected by methods of competitive adaptive reweighted sampling (CARS) and successive projections algorithm (SPA). The results showed that the accuracy of the SPA-ELM model was $94.74 \%$, which was higher than the CARS-ELM model.

Images executed by principal components analysis (PCA) were used to identify samples with defective features. Using the sobel operator and region growing algorithm, the edge and defective features of 38 Malus asiatica Nakai could be recognized and the detection accuracy was $92.11 \%$.

This study provides a theoretical reference for developing online equipment in detecting defects of Malus asiatica Nakai.

\section{Acknowledgement}

This work was supported by the National Natural Science Foundation of China (31271973) and graduate student education innovation project of Shanxi province (2017SY032). The authors are indebted to professor He Yong (College of Biosystems Engineering and Food Science, Zhejiang University, Hangzhou, China) for generously offering the hyperspectral imaging system.

\section{References}

1. .Xue Jianxin, Zhang Shujuan, Sun Haixia. Detection of Shelf Life of Malus asiatica Using Near-infrared Spectroscopy and Softening Index [J]. Transactions of the Chinese Society for Agricultural Machinery, 44 (8), 169-173 (2013)

2. Yu Keqiang, Zhao Yanru, Li Xiaoli, et al. Identification of crack features in fresh jujube using Vis/NIR hyperspectral imaging combined with image processing [J]. Computers and Electronics in Agriculture, 103,1-10 (2014)

3. Ce Yang, Won Suk Leeb, Paul Gaderc. Hyperspectral band selection for detecting different blueberry fruit maturity stage [J]. Computers and Electronics in Agriculture, 109, 23-31 (2014)

4. Zhang Baohua, Huang Wenqian, Li Jiangbo, et al. Detection of Slight Bruises on Apples Based on Hyperspectral Imaging and MNF Transform [J]. Spectroscopy and Spectral Analysis, 34 (5), 1367-1372 (2014)

5. Zhao Jiewen, Liu Jianhuan, Chen Quansheng, et al. Detecting Subtle Bruises on Fruits with Hyperspectral Imaging [J]. Transactions of the Chinese Society for Agricultural Machinery, 39 (1), 106-109 (2008)

6. Baranowski P, Mazurek W, Wozniak J, et al. Detection of early bruises in apples using hyperspectral data and thermal imaging [J]. Journal of Food Engineering, 110(3), 345-355 (2012)

7. Lee $\mathrm{W} \mathrm{H}$, Kim M S, Lee H, et al. Hyperspectral near- infrared imaging for the detection of physical damages of pear [J]. Journalof Food Engineering, 130, 1-7 (2014)

8. Lv Qiang, Tang Mingjie, Cai Jiangrong. Detection of Hidden Bruise on Kiwifruit Using Hyperspectral Imaging and Parallelepiped Classification [C] //2010 First International Conference on Cellular, Molecular Biology, Biophysics and Bioengineering (CMBB) ,309-312 (2010).

9. Byoung-Kwan Cho, Moon S. Kim, In-Suck Baek, et al. Detection of cuticle defects on cherry tomatoes using hyperspectral fluorescence imagery $[\mathrm{J}]$. Postharvest biology and 
technology, 76, 40-49 (2013)

10.Polder, G., Gerie, W.A.M., Van, D.H. Calibration and characterization of imaging spectrographs [J]. Near Infrared Spec, 11 (3), 193-210 (2003)

11. Chen Bin, Lu Bing, Lu Daoli. Parameter Optimization of Rapeseed Oil Content Model Using a Miniature Near-infrared Spectometer [J]. Modern Food Science and Technology, 31 (8), 286-292 (2015)

12. Yang Yi, Zhang Shujuan, Xue Jianxin, et al. Dynamic Discrimination of Subtly Bruised Lang Jujubes Based on Different Visible/Near-infrared Spectral Ranges [J]. Modern Food Science and Technology, 31 (8), 323-328 (2015)

13. Wu D, SunD W, He Y. Application of long-wave near infrared hyperspectral imaging for measurement of color distribution in salmon fillet [J]. Innovative Food Science \& Emerging Technologies, 16, 361-372 (2012)

14. Dora B. Heras, Francisco Argüello, Pablo Quesada-Barriuso. Exploring ELM-based spatial-spectral classification of hyperspectral images [J]. International Journal of Remote Sensing, 35 (2), 401-423. (2014)

15. Abbott J A, Lu R, Upchurch B L, et al. Technologies for non-destructive quality evaluation of fruits and vegetables [J]. Horticultural Review, 20, 1-120 (1997)

16. ElMasry G, Wang N, Vigneault C, et al. Early detection of apple bruises on different background colors using hyperspectral imaging [J]. LWT-Food Science and Technology, 41 (2), 337-345 (2008)

17. Yu K, Zhao Y, Li X, et al. Application of visible and near-infrared hyperspectral imaging for detection of defective features in Loquat [J]. Food Bioprocess Technology, 7 (11), 3077 -3087 (2014) 